\title{
Evaluation of the clinical and radiological outcome of hemi-hamate arthroplasty in the reconstruction of proximal interphalangeal Joint fracture-dislocations
}

\author{
Farid Najd Mazhar ${ }^{1}$, Mohammad Reza Bahaeddini ${ }^{1}$, Davod Jafari ${ }^{1}$, Alireza Mirzaei*1
}

\section{Abstract}

Background: Hemi-hamate arthroplasty, a new attractive method for treatment of unstable Proximal Interphalangeal (PIP) joint fracture-dislocations, offers several advantages over the previous methods. This study was designed to evaluate the clinical and radiological outcome of this procedure.

Methods: In this study, 14 patients and 15 fingers with PIP joint fracture-dislocations were evaluated, including 8 acute and 7 chronic injuries. The mean age and follow-up of the patients were 35.3 years and 29.7 months, respectively. The mean PIP joint involvement was $56.6 \%$. Objective assessment of the outcome was performed by joint alignment, motion, stability, and grip and pinch strength. Subjective evaluation of the outcome was performed using the Visual Analogue Scale (VAS) and Disabilities of the Arm, Shoulder, and Hand (DASH) score. Statistical analysis was performed using SPSS for Windows (version 16). Independent sample $t$ test or Mann-Whitney $U$ test were used for statistical comparison of the mean values. A p value of $<0.05$ was considered significant.

Results: At the final follow-up, 14 out of 15 PIP joints were clinically stable. Grip and pinch strength of the injured hand averaged $87.6 \%$ and $88 \%$ of contralateral hand, respectively. The mean PIP joint flexion, arch of motion, and flexion contracture were 82.6 , 63.6, and 19 degrees, respectively. The mean DASH score was $20.33 \pm 21.87$ in acute and $7.25 \pm 11.71$ in the chronic group ( $\mathrm{p}=0.181$ ). The mean VAS was $2.87 \pm 2.29$ in acute versus $0.42 \pm 0.78$ in chronic patients $(\mathrm{p}=0.022)$. The degenerative joint disease was observed in 5 cases.

Conclusion: Although hemi-hamate arthroplasty is a promising method for the reconstruction of severe PIP joint fracturedislocations, it is associated with minimal functional limitation and high rate of osteoarthritis.

Keywords: Proximal Interphalangeal joint, Fracture-dislocations, Hemi-hamate arthroplasty

Conflicts of Interest: None declared

Funding: None

\section{*This work has been published under CC BY-NC-SA 1.0 license.}

Copyright $\odot$ Iran University of Medical Sciences

Cite this article as: Najd Mazhar F, Bahaeddini MR, Jafari D, Mirzaei A. Evaluation of the clinical and radiological outcome of hemi-hamate arthroplasty in the reconstruction of proximal interphalangeal joint fracture-dislocations. Med J Islam Repub Iran. 2019 (26 Dec);33:152. https://doi.org/10.47176/mjiri.33.152

\section{Introduction}

High-energy traumas result in considerable destruction

Corresponding author:Dr Alireza Mirzaei, mirzaei.ar@iums.ac.ir

1. Bone and Joint Reconstruction Research Center, Shafa Orthopedic Hospital, Iran University of Medical Sciences, Tehran, Iran of bone and its associated structures (1-3). Fracture-

$\uparrow$ What is "already known" in this topic:

Fracture-dislocation of proximal interphalangeal (PIP) joint is one of the devastating and challenging injuries of the hand. Several surgical techniques have been introduced for the treatment of this injury, each having its limitations. Hemihamate arthroplasty has been introduced as a promising approach for the treatment of these injuries.

\section{$\rightarrow$ What this article adds:}

Although hemi-hamate arthroplasty provides an acceptable outcome in the majority of the cases, it is associated with minimal functional limitation and high rate of osteoarthritis. Therefore, such complications should be discussed with patients before the surgery to address the patients' expectation of the results. 
dislocation of proximal interphalangeal (PIP) joint is one of the most common, devastating, and challenging injuries of the hand that could result in persistent stiffness, pain, and angulation $(4,5)$. Therapeutic strategies mainly aim to reestablish joint congruity and stability, providing a concentric reduction, allowing early joint movement and restore normal gliding during flexion arc (6).

While open reduction and internal fixation (ORIF) (7) and extension block pining (8) could be used for less comminuted fracture types, volar plate arthroplasty (VPA) is considered as the main procedure for severely comminuted types of such fracture-dislocations for decades (9). However, each of these assays has their own limitations and disadvantages (9).

Hemi-hamate arthroplasty is considered as an attractive alternative treatment method for unstable PIP joint fracture-dislocations, which offers several advantages over the previous methods (10). Frueh et al systematically reviewed the outcome of hemi-hamate arthroplasty in the proximal interphalangeal joint reconstruction. Based on their results, although hemi-hamate arthroplasty can be considered as a reliable reconstruction approach for the treatment of severely comminuted PIP joint fracturedislocations, the number of evaluated patients is small and further investigations are needed to reach a consensus regarding its outcome, especially about the rate of postoperative osteoarthritis (11).

Hence, the aim of this study was to further evaluate the clinical and radiological outcome and rate of osteoarthritis in hemi-hamates arthroplasty of comminuted PIP joint fracture-dislocations.

\section{Methods}

\section{Patients}

This study was approved by an institutional review board (IRB) of our university under the approval code of 1748 , and informed consent was obtained from each patient. In a prospective study, all patients who were referred to our center between 2011 and 2015 with a PIP joint fracture-dislocation were evaluated for eligibility criteria. A total of 61 patients with 63 PIP joint fracture-dislocations were treated in our center during the study period. A total of 30 PIP joint was managed with ORIF through a shot- gun approach, the results of which were published in the European volume of the Journal of Hand Surgery (12). The severity of comminution led to the application of hemi-hamate arthroplasty for the remaining 33 PIP joint fracture-dislocations from 31 patients. The inclusion criteria were described as the involvement of at least $40 \%$ of the volar middle phalangeal surface, injuries not amenable by fixation, and a minimum follow-up of 6 months. Considering our center as the main referral center of the hand surgery, patients were referred from all over the country, and some patients living in distant areas were not accessible. Due to this problem, 10 patients were lost to followup and were omitted from the study. Also, 2 patients were followed-up for fewer than 6 months and were excluded from the study. From the remaining 19 patients, 14 agreed to enter the study, which included 8 acute and 7 chronic injuries. Those cases referred at fewer than 6 weeks of injury were considered as acute.

Review of the demographic, clinical, and radiographic information of the patients was performed through their medical files and final visits. The mean age of the patients was 35.3 years, ranging from 22 to 50 years. The mean follow-up of the patients was 29.7 months, ranging from 7 to 55 months. The mean PIP joint involvement was $56.6 \%$. The mean interval from the injury to the treatment was 11.37 days for acute injuries, ranging from 4 to 26 days, and 127.1 days for the chronic type, ranging from 63 to 230 days. Falling, direct trauma, and fighting were the most common mechanisms of PIP joint fracturedislocations (Table 1).

Subjective functional outcomes were assessed by Disabilities of the Arm, Shoulder, and Hand (DASH) outcome measure in Persian (13) and Visual Analogue Scale (VAS) scoring system (14). Objective assessment of the outcome included joint alignment, motion, grip strength, osteonecrosis, fracture union, resorption, collapse, joint subluxation, and degenerative joint disease. All the assessments were performed by a fellowshiptrained hand surgeon who was not engaged in the treatment of patients. Anterior/posterior (AP) and lateral Xrays were used for the radiological evaluation of the outcome.

\begin{tabular}{|c|c|c|c|c|c|c|c|c|c|}
\hline $\begin{array}{l}\text { Case } \\
\text { Number }\end{array}$ & $\begin{array}{l}\text { Age } \\
\text { (Year) }\end{array}$ & Gender & $\begin{array}{l}\text { Involved } \\
\text { Finger }\end{array}$ & $\begin{array}{l}\text { Dominant } \\
\text { hand }\end{array}$ & $\begin{array}{c}\text { Joint } \\
\text { Involvement (\%) }\end{array}$ & $\begin{array}{l}\text { Acute/ch } \\
\text { ronic }\end{array}$ & $\begin{array}{l}\text { Average time } \\
\text { to surgery (day) }\end{array}$ & $\begin{array}{l}\text { Follow-up } \\
\text { (Month) }\end{array}$ & $\begin{array}{l}\text { Mechanism } \\
\text { of injury }\end{array}$ \\
\hline 1 & 28 & Male & Right $3^{\text {rd }}$ & Right & $50 \%$ & Chronic & 230 & $41 \mathrm{~m}$ & MVA* \\
\hline 2 & 34 & Male & Left $5^{\text {th }}$ & Right & $60 \%$ & Acute & 4 & $33 \mathrm{~m}$ & Falling \\
\hline 3 & 50 & Male & Left $4^{\text {th }}$ & Right & $60 \%$ & Acute & 15 & $36 \mathrm{~m}$ & Contact Sport \\
\hline 4 & 43 & Male & Right $3^{\text {rd }}$ & Right & $50 \%$ & Chronic & 63 & $55 \mathrm{~m}$ & MVA* \\
\hline 5 & 44 & Male & Left $3^{\text {rd }}$ & Left & $50 \%$ & Acute & 5 & $47 \mathrm{~m}$ & Fighting \\
\hline 5 & 44 & Male & Left $4^{\text {th }}$ & Left & $50 \%$ & Acute & 5 & $47 \mathrm{~m}$ & Fighting \\
\hline 7 & 22 & Male & Left $3^{\text {rd }}$ & Right & $70 \%$ & Chronic & 184 & $36 \mathrm{~m}$ & Falling \\
\hline 8 & 28 & Male & Right $3^{\text {rd }}$ & Right & $70 \%$ & Chronic & 175 & $31 \mathrm{~m}$ & Direct trauma \\
\hline 9 & 24 & Male & Left $3^{\text {rd }}$ & Right & $50 \%$ & Acute & 26 & $25 \mathrm{~m}$ & Fighting \\
\hline 10 & 30 & Male & Right $3^{\text {rd }}$ & Right & $40 \%$ & Chronic & 100 & $9 \mathrm{~m}$ & Direct trauma \\
\hline 11 & 49 & Male & Left $5^{\text {th }}$ & Right & $70 \%$ & Acute & 6 & $30 \mathrm{~m}$ & Direct trauma \\
\hline 12 & 29 & Male & Right $4^{\text {th }}$ & Left & $40 \%$ & Acute & 14 & $11 \mathrm{~m}$ & Falling \\
\hline 13 & 36 & Female & Left $5^{\text {th }}$ & Left & $70 \%$ & Acute & 16 & $8 \mathrm{~m}$ & Direct trauma \\
\hline 14 & 24 & Male & Left $3^{\text {rd }}$ & Right & $50 \%$ & Chronic & 68 & $7 \mathrm{~m}$ & Falling \\
\hline
\end{tabular}




\section{Surgical technique}

The protocol for the treatment of a volar based fracturedislocation of PIP joint in our hand surgery department was as follows: When the injury was not amenable by conservative treatment or extension block pining, we proceed to treat the injury by open reduction under general anesthesia. The surgical approach was performed as previously described with some minor modifications (15). Accordingly, through a U-shaped incision, radial or ulnar based flap was raised on PIP joint (radial based flap for the second and third fingers and ulnar-based flap for the fourth and fifth fingers to reduce the incision site sensitivity in the contact area), the flexor tendon sheet was opened between A2 and A4 pulleys and a radial or ulnar-based flap, including A3 pulley, was elevated. Subsequently, the flexor tendons were retracted and volar plate was exposed. After separation of the attached fracture fragments, the volar plate was bended proximally to expose the proximal phalangeal head and the middle phalangeal base. Finally, the collateral ligaments were released from their origin for the completion of "Shotgun" approach by hyperextension of the PIP joint (Fig. 1). At this step, we evaluated the fracture fragments pattern. If it was possible to fix the fracture by screws, it was selected as the therapeutic approach of choice. When the fracture was much comminuted, we shifted the therapeutic approach to the hemihamate procedure. The fracture site was then prepared for the placement of hamate autograft using a mini oscillating saw. In the next step, a 3-4 cm longitudinal incision was made on the carpometacarpal joint at the bases of the ring and small fingers, preserving the dorsal sensory branches of the ulnar nerve. After a dorsal capsulotomy, once the hamate was visualized, the graft dimensions were marked and the hamate cut was performed. Surprisingly, during our procedures, we noticed the inconstant anatomy and shape of the distal hamate bone. The height of the middle ridge of the distal hamate and dorsal slope were not equal, and in some cases, the middle ridge was very short. We harvested the graft slightly larger than needed and trimmed it by using mini-oscillating saw and small rongeur to fit the recipient area. The hamate autograft was then placed into the defect at the base of the middle phalanx. Provisional fixation was performed by placing two $0.8-\mathrm{mm} \mathrm{K}$-wires in the peripheral part of the graft after fine and proper trimming. Subsequently, the graft was fixed to the intact dorsal cortex of the middle phalanx using 2 screws (1.5 mm) (Fig. 2a). The joint was relocated afterward, and the proper screw length and graft position were assessed with lateral and anteroposterior X-rays (Fig. 2b). After reattachment of the volar plate to the stumps of the collateral ligaments and the lateral margins of the middle phalanx, final clinical evaluation was performed to ensure an acceptable range of motion and stability. Subsequently, the reflected flexor sheet was interposed to act as a protective layer between the volar plate and flexor tendons. The incision was closed afterwards. For one of our patients (Case no5) who had injuries of the left third and fourth fingers, we used the contralateral hamate and the ipsilateral hamate autograft. All surgeries were performed by 1 surgeon (F.N.M) who used the same surgical ap-

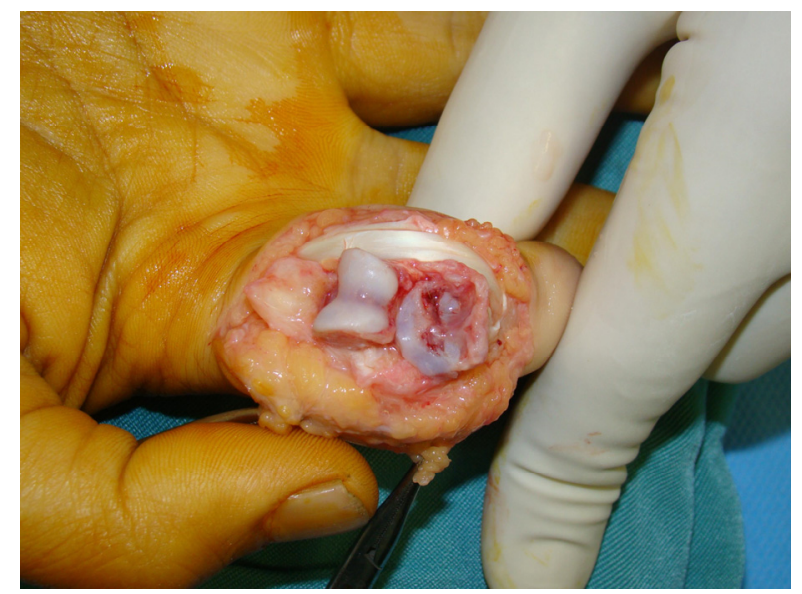

Fig. 1. Shot-gun approach was completed to expose the PIP joint. The fracture was much comminuted and fixation was not feasible.
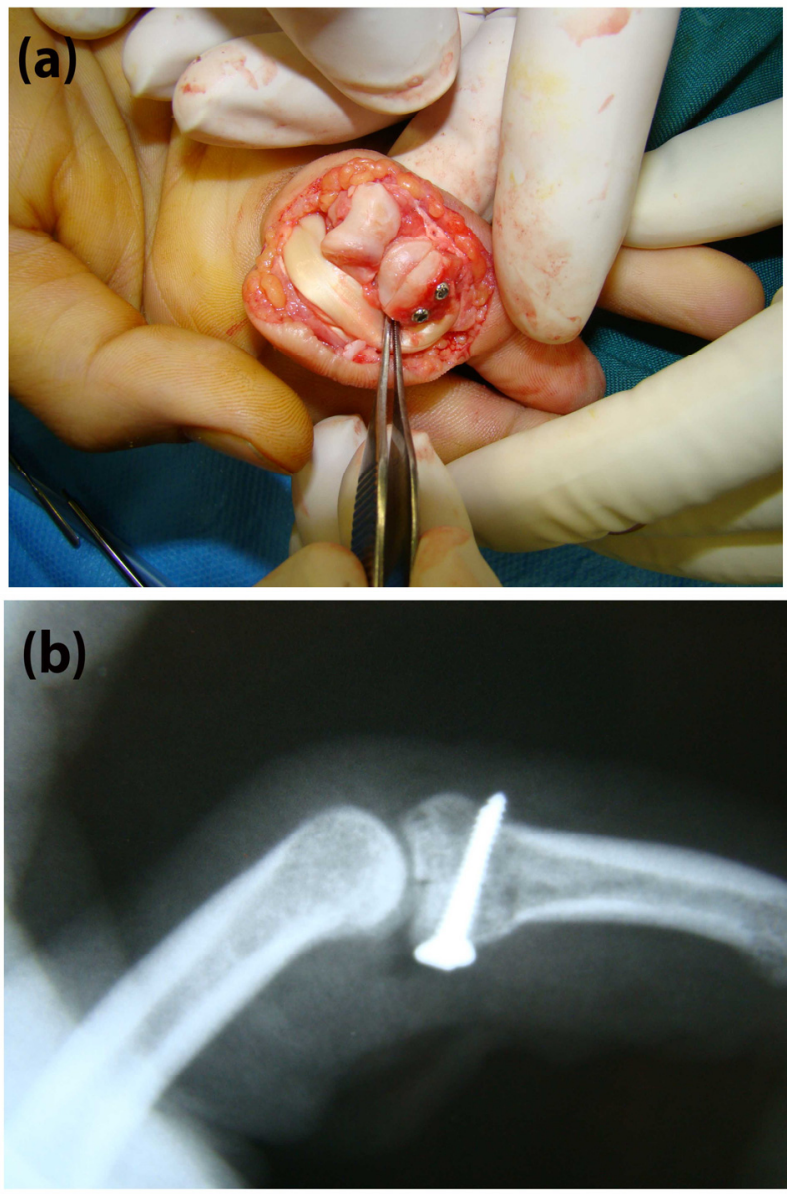

Fig. 2. (a) The Hemi hamate autograft was fixed by two mini screws in the prepared box; (B) Portable X-ray to evaluate the reduction and fixation quality.

proach and postoperative protocol in all cases.

\section{Postoperative protocol}

A dorsal splint was used for 3 weeks, with PIP joint held in approximately $20^{\circ}$ of flexion to protect the volar plate repair. Nearly one day after the surgery, the active and gentle passive range of motion exercises were initiated as tolerated. Physiotherapy was started 3 weeks after the operation to achieve the optimal range of motion 
of all small joints. In the first postoperative 3 weeks, weekly radiographic and clinical evaluation were performed to ensure that the patient was following the instructions and has not lost the reduction.

\section{Statistical analysis}

Statistical analysis was performed using SPSS for Windows (version 16). Central tendency and variability for numeric variables were measured using the mean and standard deviation (SD), respectively. Independent sample $\mathrm{t}$ test (for parametric variables) and Mann-Whitney U test (for nonparametric variables) were used for statistical evaluations. A $\mathrm{p}$ value of $<0.05$ was considered significant.

\section{Results}

At the latest follow-up session, except one PIP joint, which was complicated by dorsal subluxation, all other PIP joints were clinically stable to coronal and sagittal stress. Table 2 demonstrates the outcome measures of our cohort in detail. In total, 9 third fingers, 3 forth fingers, and 3 fifth fingers were involved.

The mean pinch strength was $20.3 \mathrm{Ib} \pm 4.5$ and $17.7 \mathrm{Ib} \pm 4.7$ for the healthy and injured hands, respectively. Accordingly, the pinch strength of the injured hand averaged $88 \%$ of contralateral hand, ranging from $66.6 \%$ to $120 \%$.

The mean grip strength was $93.9 \mathrm{Ib} \pm 12.3$ and $82 \mathrm{Ib} \pm 17.4$ for the healthy and injured hands, respectively. Accordingly, the grip strength of the injured hand averaged
$87.6 \%$ of the contralateral hand, ranging from $67.9 \%$ to $105 \%$.

The mean PIP joint flexion, arch of motion, and flexion contracture was $82.6^{\circ} \pm 20.9^{\circ}, 63.6^{\circ} \pm 23.8^{\circ}$, and $19^{\circ} \pm 19.6^{\circ}$, respectively. The mean distal interphalangeal joint (DIP) flexion, DIP arch of motion, and DIP flexion contracture was $59.6^{\circ} \pm 19.1^{\circ}, 59.6 .6^{\circ} \pm 19.1^{\circ}$, and $0.0^{\circ}$, respectively. The mean metacarpophalangeal joint (MCP) flexion, MCP arch of motion, and MCP flexion contracture was $90^{\circ} \pm 0.0^{\circ}, 90^{\circ} \pm 0.0^{\circ}$, and $0.0^{\circ}$, respectively (Figs. 3a, b, and c). The mean DASH score was $13.56 \pm 18.47$, and the mean VAS was $1.73 \pm 2.12$ (Table 3 ).

DASH score was higher in acute PIP joint fractureinjuries. In this regard, the mean DASH score was $20.33 \pm 21.87$ in acute and $7.25 \pm 11.71$ in the chronic group. This difference was not statistically significant $(\mathrm{p}=0.181)$. VAS was significantly higher in the acute group compared to chronic PIP joint injuries $(\mathrm{p}=0.022)$, with a mean value of $2.87 \pm 2.29$ versus $0.42 \pm 0.78$, respectively.

DASH score and VAS was considerably higher in 2 patients (Cases 13 and 3). Patient number 13, with a DASH score of 64.67 and VAS of 8, was a young lady with depression and anxiety disorder, who was being treated by a psychiatrist. Patient number 3, with a DASH score of 38.15 and VAS of 3 , had exceptionally higher scores without any clear reason. After excluding these 2 patients from the statistical analysis, the mean VAS and DASH score changed to $1.15 \pm 1.21$ and $7.75 \pm 9.71$, respectively. However, these values were still higher in acute injuries,

\begin{tabular}{|c|c|c|c|c|c|c|c|c|c|c|c|}
\hline Variable & 1 & 2 & 3 & 4 & $5\left(3^{\text {rd }}\right)$ & $5\left(4^{\text {th }}\right)$ & 6 & 7 & 8 & 9 & 10 \\
\hline Pinch strength, healthy hand (Ib) & 24 & 25 & 12 & 20 & 18 & 18 & 16 & 25 & 20 & 25 & 15 \\
\hline Pinch strength, injured hand (Ib) & 18 & 17 & 10 & 24 & 12 & 12 & 14 & 18 & 23 & 20 & 15 \\
\hline Grip strength, healthy hand (Ib) & 110 & 105 & 85 & 105 & 75 & 75 & 80 & 85 & 100 & 105 & 95 \\
\hline Grip strength, injured hand (Ib) & 95 & 90 & 64 & 110 & 65 & 65 & 100 & 65 & 105 & 90 & 70 \\
\hline Contralateral grip strength (\%) & 86.3 & 85.7 & 75.3 & 104.7 & 86.6 & 86.6 & 125 & 76.4 & 105 & 85.7 & 73.6 \\
\hline PIP flexion contracture $\left({ }^{\circ}\right)$ & 30 & 5 & 30 & $\mathrm{~N}$ & $\mathrm{~N}$ & $\mathrm{~N}$ & 30 & 30 & 20 & 30 & 30 \\
\hline DIP flexion $\left({ }^{\circ}\right)$ & 70 & 70 & 30 & 70 & 70 & 70 & 10 & 70 & 70 & 70 & 70 \\
\hline DIP Arch of motion $\left({ }^{\circ}\right)$ & 70 & 70 & 30 & 70 & 70 & 70 & 10 & 70 & 70 & 70 & 70 \\
\hline DIP flexion contracture $\left({ }^{\circ}\right)$ & $\mathrm{N}$ & $\mathrm{N}$ & $\mathrm{N}$ & $\mathrm{N}$ & $\mathrm{N}$ & $\mathrm{N}$ & $\mathrm{N}$ & $\mathrm{N}$ & $\mathrm{N}$ & $\mathrm{N}$ & $\mathrm{N}$ \\
\hline MCP flexion $\left({ }^{\circ}\right)$ & 90 & 90 & 90 & 90 & 90 & 90 & 90 & 90 & 90 & 90 & 90 \\
\hline
\end{tabular}
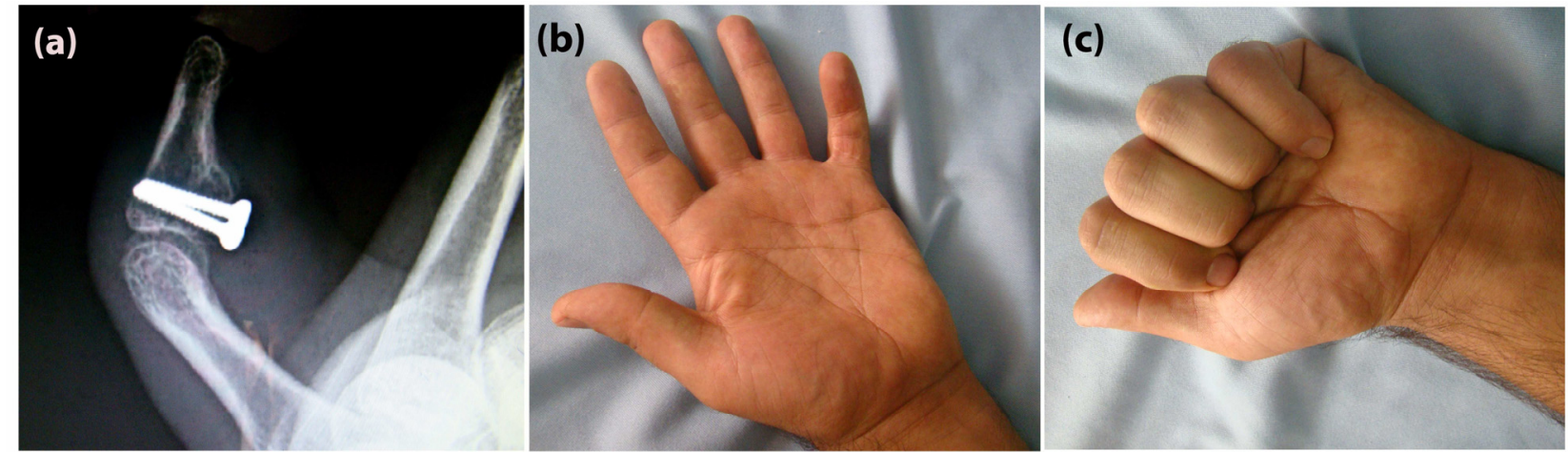

Fig. 3. (a) Radiographic outcome of hemi-hamate arthroplasty 11 months after the surgery (Case No 12); (b) range of motion in extension; (c) range of motion in flexion. 


\begin{tabular}{|c|c|c|c|c|c|c|}
\hline Variable & $\mathrm{N}$ (Fingers) & $\mathrm{N}$ (Patients) & Minimum & Maximum & Mean & Std. Deviation \\
\hline $\begin{array}{l}\text { Pinch strength (Healthy hand) (Ib) } \\
\text { (Ib }\end{array}$ & 15 & 14 & 12 & 27.00 & 20.33 & 4.54 \\
\hline Pinch strength (Injured hand) (Ib) & 15 & 14 & 10 & 25 & 17.73 & 4.72 \\
\hline Grip strength (Healthy hand) (Ib) & 15 & 14 & 75 & 110 & 93.93 & 12.37 \\
\hline Grip strength (Injured hand) (Ib) & 15 & 14 & 60 & 110 & 82.00 & 17.48 \\
\hline PIP flexion $\left({ }^{\circ}\right)$ & 15 & 14 & 30 & 110 & 82.00 & 20.51 \\
\hline PIP Arch of motion $\left({ }^{\circ}\right)$ & 15 & 14 & 30 & 110 & 63.66 & 23.86 \\
\hline PIP flexion contracture $\left({ }^{\circ}\right)$ & 15 & 14 & .00 & 70 & 18.33 & 20.14 \\
\hline DIP flexion $\left({ }^{\circ}\right)$ & 15 & 14 & 10 & 70 & 59.66 & 19.12 \\
\hline DIP Arch of motion $\left({ }^{\circ}\right)$ & 15 & 14 & 10 & 70 & 59.66 & 19.12 \\
\hline DIP flexion contracture $\left({ }^{\circ}\right)$ & 15 & 14 & .00 & .00 & .00 & .00 \\
\hline MCP flexion $\left({ }^{\circ}\right)$ & 15 & 14 & 90 & 90 & 90.00 & .00 \\
\hline MCP Arch of motion $\left({ }^{\circ}\right)$ & 15 & 14 & 90 & 90 & 90.00 & .00 \\
\hline MCP flexion contracture $\left({ }^{\circ}\right)$ & 15 & 14 & .00 & .00 & .00 & .00 \\
\hline DASH score & 15 & 14 & .00 & 64.47 & 13.56 & 18.47 \\
\hline VAS & 15 & 14 & .00 & 8 & 1.73 & 2.12 \\
\hline
\end{tabular}
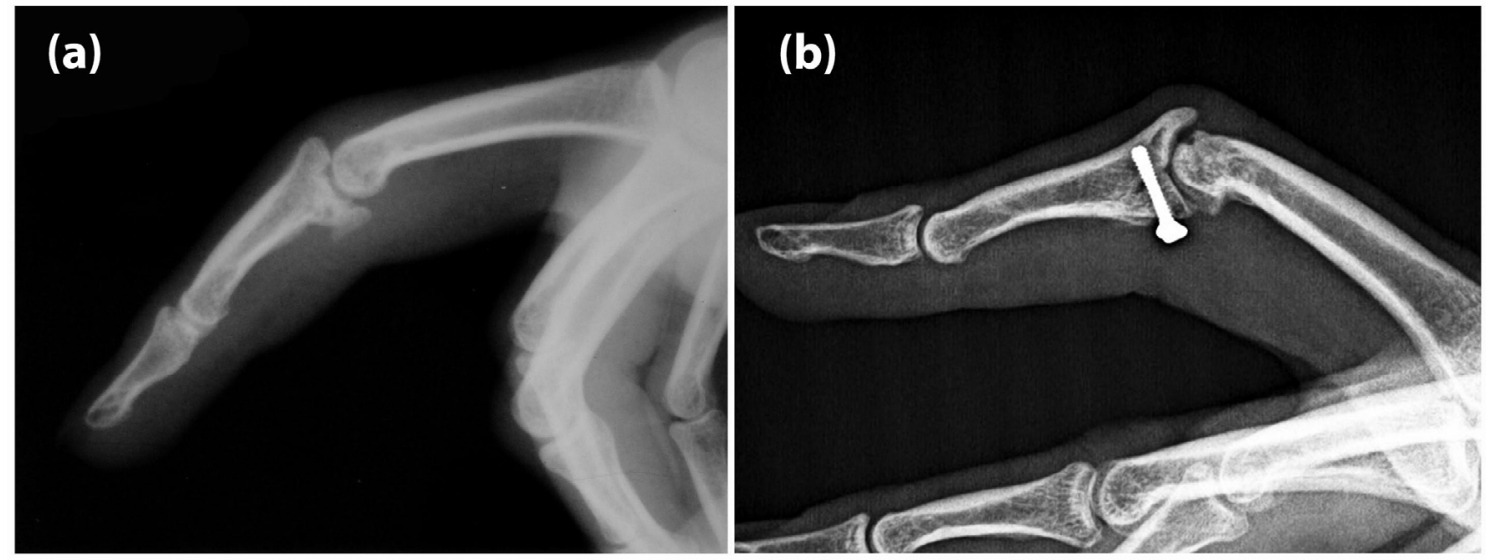

Fig. 4. (a) Preoperative X-ray of a chronic PIP joint fracture dislocation (Case No 1); (b) Degenerative joint disease 41 months after hemi-hamate arthroplasty (case No 1). Surgery was performed 230 days after injury.

with the mean DASH score and VAS of 10 and 2 in acute and 5.8 and 0.42 in chronic cases.

No significant differences were observed in objective outcome measures between acute and chronic injuries. Moreover, no significant correlation was observed between the level of involvement and outcome measures such as DASH, VAS, etc.

Complications: At the final follow-up session, a complete union was observed in all injured PIP joints of our cohort. No case of infection was observed in our patients. Graft resorption and PIP joint subluxation were seen in one case of old injury (case N0 7). Degenerative joint disease (DJD) was observed in 5 cases, but this was not associated with impairment of function, and no further treatment was proposed for those patients (Fig. 4a, b). One of our patients suffered from constant paresthesia along the dorsal aspect of the fifth finger. However, he refused our treatment plan of release for ulnar nerve dorsal sensory branch.

\section{Discussion}

Based on the result of this study, hemi-hamate arthroplasty provides a grip and pinch strength equivalent to $87.6 \%$ and $88 \%$ of the contralateral hand. Furthermore, an acceptable PIP joint flexion, arch of motion, and flexion contracture could be achieved following this procedure.
The subjective measures were also at an acceptable range, suggesting hemi-hamate arthroplasty as a promising method for the reconstruction of severe PIP joint fracturedislocations.

Literature review reveals only a few studies about the clinical and radiographic outcomes of hemi-hamate arthroplasty $(11,15,16)$. Williams et al reported a series of 13 patients, with a mean defect of $60 \%$, who were treated by hemi-hamate arthroplasty. They reported a mean PIP joint motion of $85^{\circ}$ and grip strength of $80 \%$ of the uninjured side. They also observed 2 recurrent dorsal subluxations, while the osseous union was observed in all of their patients at a mean follow-up of 16 months (15).

Calfee et al also reviewed a series of 22 patients with $50 \%$ joint surface involvement who underwent hemihamate arthroplasty, with a mean follow-up of 4.5 years. According to their report, 14 patients had acute injuries (fewer than 6 weeks), while other 8 patients were referred with chronic injuries. They reported an active PIP joint motion of $70^{\circ}$ and contracture of $19^{\circ}$, with an average grip strength of $95 \%$ of the opposite hand. VAS score was 2.6 for chronic and 1.4 for acute reconstructions. Besides, the DASH score reported being 9 for chronic and 2 for acute cases (16).

Burnier et al reviewed the clinical and radiological results after hemi-hamate resurfacing arthroplasty in 19 pa- 
tients with $40 \%$ joint surface involvement. They reported a mean active PIP joint arch of motion of $83^{\circ}$, a mean VAS of 1.1 and a mean DASH score of 11 . According to their report, the mean pinch strength was $82 \%$ of the opposite side (17).

Afendras et al evaluated the radiographic outcome of hemi-hamate arthroplasty after a minimum follow-up of 4 years with special reference to the development of osteoarthritis and its relation to clinical symptoms in 8 patients. They reported a mean active PIP joint arch of motion of $67^{\circ}$, with grip strength of $91 \%$ of the uninjured side, and a mean VAS of 1 . Two cases of severe arthritis and 2 cases of mild arthritis were observed in their patients. However, only one case had troublesome pain. Based on their report, although the hemi-hamate arthroplasty is an attractive alternative to other surgical options, osteoarthritis may develop in some cases in the mid-term (18).

In total, the results of the present study were in accordance with those of earlier investigations. In the present study, the mean PIP joint arch of motion was $63.6^{\circ}$ and the mean PIP jont contracture was $19^{\circ}$. The grip strength of the injured hand averaged $87.6 \%$ of the opposite hand. Also, 5 cases $(26.3 \%)$ of osteoarthritis were found in our patients.

We believe that the limited number of patients could be regarded as the major limitation of this study that could have affected the power of statistical analysis. Thus, further studies with larger sample size are warranted. Besides, longer follow-ups are needed to more precisely evaluate the long-term outcome of hemi-hamate arthroplasty.

\section{Conclusion}

Although the result of hemi-hamate arthroplasty revealed to be promising for the reconstruction of severe PIP joint fracture-dislocations, at least in the short-term follow-up, minimal functional limitation was obtained in the majority of the cases. In addition, osteoarthritis of PIP joint might develop in a considerable number of patients. Therefore, patients' expectations should be addressed by providing this information preoperatively.

\section{Conflict of Interests}

The authors declare that they have no competing interests.
7. Freeland AE, Benoist LA. Open reduction and internal fixation method for fractures at the proximal interphalangeal joint. Hand Clin. 1994;10(2):239-50.

8. Bear D, Weichbrodt M, Huang C, Hagberg W, Balk M. Unstable dorsal proximal interphalangeal joint fracture-dislocations treated with extension-block pinning. Am J Orthop (Belle Mead, NJ). 2015;44(3):122-6.

9. Freiberg A, Pollard BA, Macdonald MR, Duncan MJ. Management of proximal interphalangeal joint injuries. Hand Clin. 2006;22(3):235-42.

10. Hastings H, Capo J, Steinberg B, Stern P, editors. Hemicondylar hamate replacement arthroplasty for proximal interphalangeal joint fracture/dislocations (abstr). 54th Annual Meeting of the American Society for Surgery of the Hand, Boston, MA; 1999.

11. Frueh FS, Calcagni M, Lindenblatt $\mathrm{N}$. The hemi-hamate autograft arthroplasty in proximal interphalangeal joint reconstruction: a systematic review. J Hand Surg Eur Vol. 2015 Jan;40(1):24-32.

12. Najd Mazhar F, Jafari D, Taraz H, Mirzaei A. Treatment of dorsal fracture-dislocations of the proximal interphalangeal joint using the shotgun approach. J Hand Surg Eur Vol. 2018;43(5):499-505.

13. Mousavi SJ, Parnianpour M, Abedi M, Askary-Ashtiani A, Karimi A, Khorsandi A, et al. Cultural adaptation and validation of the Persian version of the Disabilities of the Arm, Shoulder and Hand (DASH) outcome measure. Clin Rehab. 2008;22(8):749-57.

14. Crichton N. Visual analogue scale (VAS). J Clin Nurs. 2001;10(5):706-6.

15. Williams RM, Kiefhaber TR, Sommerkamp TG, Stern PJ. Treatment of unstable dorsal proximal interphalangeal fracture/dislocations using a hemi-hamate autograft. J Hand Surg Am. 2003;28(5):856-65.

16. Calfee R, Kiefhaber T, Sommerkamp T, Stern P. Hemi-hamate arthroplasty provides functional reconstruction of acute and chronic proximal interphalangeal fracture-dislocations. J Hand Surg Am. 2009;34(7):1232-41.

17. Burnier M, Awada T, Braun FM, Rostoucher P, Ninou M, Erhard L. Treatment of unstable proximal interphalangeal joint fractures with hemi-hamate osteochondral autografts. J Hand Surg Eur Vol. 2017 Feb;42(2):188-193

18. Afendras G, Abramo A, Mrkonjic A, Geijer M, Kopylov P, Tägil M. Hemi-hamate osteochondral transplantation in proximal interphalangeal dorsal fracture dislocations: a minimum 4 year followup in eight patients. J Hand Surg Eur Vol. 2010;35(8):627-31.

\section{References}

1. Mazhar FN, Jafari D, Mirzaei A. Evaluation of functional outcome after nonsurgical management of terrible triad injuries of the elbow. $\mathrm{J}$ Shoulder Elbow Surg. 2017;26(8):1342-7.

2. Mazhar FN, Ebrahimi H, Jafari D, Mirzaei A. Radial head resection versus prosthetic arthroplasty in terrible triad injury: a retrospective comparative cohort study. Bone Joint J. 2018;100(11):1499-505.

3. Mazhar FN, Jafari D, Jafari SS, Mirzaei A. Ligament Reconstruction of Traumatic Pure Dislocation of Carpometacarpal Joint of the Thumb: A Series of Acute and Chronic Cases. J Hand Microsurg. 2018. DOI: $10.1055 / \mathrm{s}-0038-1669367$

4. Kang R, Stern PJ. Fracture dislocations of the proximal interphalangeal joint. J Am Soc Surg Hand. 2002;2(2):47-59.

5. Bindra R, Woodside JC. Treatment of Proximal Interphalangeal Joint Fracture-Dislocations. JBJS Rev. 2015;3(12):e1.

6. Calfee R, Sommerkamp T. Fracture-dislocation about the finger joints. J Hand Surg Am. 2009;34(6):1140-7. 